\title{
The age-metallicity relation in the thin disk
}

\author{
Ji Li, Bo Liang and Weishi Fan \\ College of Physics and Information Engineering, Hebei Normal University, Shijiazhaung \\ 050016, P. R. China \\ email: liji@mail.hebtu.edu.cn
}

\begin{abstract}
With two stellar sample A and B, the age-metallicity relation (AMR) in the Galactic thin disk is investigated. The results show two different AMRs: one is a nearly flat AMR from photometric analysis of sample A, the other is an obvious AMR derived from spectroscoipic analysis of sample B.
\end{abstract}

Keywords. Galaxy: solar neighbourhood, Galaxy: disk, Galaxy: kinematics and dynamics, Galaxy: evolution, Stars: fundamental parameters

\section{Introduction}

Many studies have found there is a clear relation between the ages and the metallicities of the solar neighbourhood disk stars (e.g. Twarog 1980; Meusinger et al. 1991; Ng \& Bertelli 1998; Rocha-Pinto et al. 2000). In contrast to this Edvardsson et al. (1993) found no particular evidence for an AMR in the solar neighbourhood and other two large sample investigations(Feltzing et al. 2001(F01), Nordström et al. 2004(N04)) confirmed this.

Recently, Bensby et al. (2004) investigated a sample of 229 nearby thick disk stars and found there is indeed an AMR in the thick disk. Such a clear AMR is also confirmed by Haywood (2006). The question then arises: could it be so that the lack of a relation between ages and metallicities for stars in the solar neighbourhood is in fact a population effect? Is there a similar AMR in the thin disk as that in the thick disk? In the study presented here we will address the question of a relation between ages and metallicities for stars that are kinematically selected to resemble the thin disk closely.

\section{Stellar sample}

To obtain an accurate and reliable AMR, a comprehensive and unbiased stellar sample is important. Our stellar sample includes two samples. Sample A consists of 4007 nearby stars selected from the common stars of two large sample works F01 and N04 by requiring the common stars showing consistent ages within the difference of $\pm 3 \mathrm{Gyr}$. Sample B consists of 641 stars from 13 spectroscopic works (Edvardsson et al. (1993), Nissen \& Schuster 1997, Jehin et al.1999, Fulbright 2000, Mishenina \& Kyukh 2001, Reddy et al. 2003, Reddy et al. 2006, Bensby et al. 2005, Grraton et al. 2003, Jonsell et al. 2005, Brewer et al. 2006, Fuhrmann et al. 2004, Gehren et al. 2004).

\section{Results}

The population membership of the sample stars were determined with pure kinematical criteria(Bensby et al. 2004). Figure 1 shows the AMR for 3856 thin disk stars from sample A, where the ages are the average values corresponding to that from $\mathrm{F} 01$ and $\mathrm{N} 04,[\mathrm{Fe} / \mathrm{H}]$ are photometric metallicities from N04. Figure 2 shows the AMR of 434 thin disk stars 

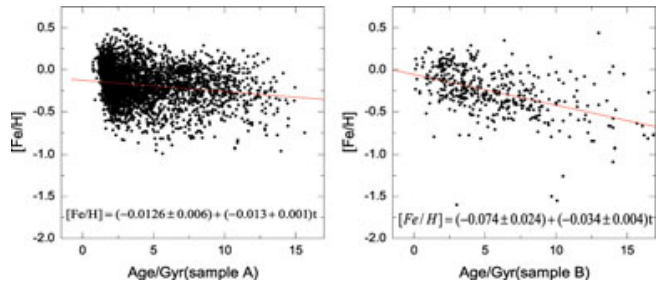

Figure 1. AMR in thin disk stars.

from sample B, where the stellar ages are our re-calculations using $\mathrm{Y}^{2}$-isochrones (Yi et al. $2003),[\mathrm{Fe} / \mathrm{H}]$ are the spectroscopic metallicities adopted from the original references.

\section{Discussion and conclusion}

Figure 1 shows there is an age-metallicity relation present in the Galactic thin disk either for sample A or sample B, but the AMR derived from sample B is more declining than that derived from sample A. This result means that the AMR of stars in the thin disk suffering from a significant population effect. To fully understand the inward nature of the AMR in thin disk, more complete and accurate observations are needed, and the methods used to compute stellar ages and their uncertainties, as well as the choice and verification of stellar models are far more urgent issues at present.

\section{Acknowledgements}

Supported by the National Natural Science Foundation under grant number 10778616.

\section{References}

Bensby, T., Feltzing S., \& Lundström I. 2004, A\&A 421, 969

Bensby, T., Feltzing S., \& Lundström I., et al. 2005, A\& A 433, 185

Brewer, Mary-Margaret \& Carney Bruce W. 2006, AJ 131, 431

Edvardsson, B., Andersen, J., Gustafsson, B., et al. 1993 A\&A 275, 101

Feltzing, S., Holmberg, J., \& Hurley J. R. 1983, A\& A 377, 911

Fuhrmann, K. 2004, AN 325, 3

Fulbright, J. P. 2000, AJ 120, 1841

Gehren, T., Liang, Y. C., \& Shi, J. R. et al. 2004, A\&A 413, 1045.

Gratton, R. G, Carretta E., \& Claudi R., et al. 2003, A\&A 404, 187

Haywood, M. 2006, MNRAS 371, 1760

Jehin, E., Magain, P., \& Neuforge, C., et al. 1999, A\&A 341, 241

Jonsell, K., Edvardsson, B., \& Gustafsson, B., et al. 2005, A\&A 440, 321

Meusinger, H., Srecklum, B., \& Reimann, H-G. 1991, A\&A 245, 57

Mishenina, T. V. \& Kovtyukh, V. V. 2001, A\& A 370, 951

Ng Y. K. \& Bertelli, G. 1998, A\& A 329, 943

Nordström B., Mayor M., \& Andersen, J., et al. 2004, A\&A 418, 989

Reddy, B. E., Tomkin, J., \& Lambert, D. L., et al. 2003, MNRAS 340, 304

Reddy, B. E., Lambert, D. L., \& Prieto, C. A. 2006, MNRAS 367, 1329

Rocha-Pinto, H. J., Maciel, W. J., \& Scalo, J., et al. 2000, A\&A 358, 850

Twarog, B. A. 1980, ApJS 44, 1

Yi, S., Demarque, P., \& Kim, Y. C., et al. 2003, ApJS 144, 256 